\title{
CHANGES IN SEVERAL INFLAMMATORY AND OXIDATION MARKERS AFTER OVALBUMIN-SENSITIZATION IN A GUINEA PIG MODEL OF ALLERGIC ASTHMA - A PILOT STUDY
}

\author{
Mokra $\mathrm{D}^{1}$, Drgova $\mathrm{A}^{2}$, Pullmann R sr. ${ }^{3}$, Mikolka $\mathrm{P}^{1}$, Antosova $\mathrm{M}^{4}$, Mokry $\mathrm{J}^{4}$
}

\begin{abstract}
${ }^{1}$ Department of Physiology, Jessenius Faculty of Medicine, Comenius University, Martin ${ }^{2}$ Department of Medical Biochemistry, Jessenius Faculty of Medicine, Comenius University, Martin

${ }^{3}$ Department of Clinical Biochemistry, Jessenius Faculty of Medicine, Comenius University and University Hospital, Martin

${ }^{4}$ Department of Pharmacology, Jessenius Faculty of Medicine, Comenius University, Martin, Slovakia
\end{abstract}

\begin{abstract}
A b s t r a t
Ovalbumin (OVA)-sensitization is a common way to evoke changes similar to changes in allergic asthma in humans. Activated cells produce various pro-inflammatory and vasoactive substances including reactive oxygen species. The goal of this pilot study was to evaluate mobilization of leukocytes into the lungs and oxidation processes induced by OVA-sensitization in a guinea-pig model of allergic asthma. Guinea-pigs were divided into OVAsensitized and naïve animals. After sacrificing animals, blood samples were taken and total and differential leukocyte counts were calculated, and eosinophil cationic protein (ECP) and total antioxidant status (TAS) in the plasma were determined. Left lungs were saline-lavaged and total number of cells and differential leukocyte count in the bronchoalveolar lavage fluid (BAL) were calculated. Right lung tissue was homogenized, ECP, TAS and products of lipid and protein oxidation (thiobarbituric acid-reactive substances and lysine-lipoperoxidation end-products) were determined in the lung homogenate. OVA-sensitization increased a total number of cells and percentages of eosinophils and neutrophils and slightly increased ECP in the blood and in the BAL fluid. In addition, increased lipid and protein oxidation in the lung homogenate, and decreased TAS in the plasma was found in OVAsensitized compared to naïve animals. In conclusion, OVA-sensitization increased mobilization of leukocytes into the lungs and elevated production of reactive oxygen spesies (ROS), accompanied by a decrease in plasma TAS.
\end{abstract}

Key words: allergic asthma, ovalbumin-sensitization, oxidation stress, reactive oxygen species, guinea pig

\section{INTRODUCTION}

Allergic asthma is a respiratory disease with a complex pathophysiology. It is characterized by airway hyperresponsiveness, increased irritability of sensory nerves in the airways and enhanced mucus secretion, as well as by acute and chronic inflammation, edema and airway remodeling (1). Activation of eosinophils, neutrophils, T-lymphocytes and mast cells is associated with increased production of reactive oxygen species (ROS) and reduced antioxidant status, as it has been described $(2,3)$. ROS, such as superoxide anion, hydrogen peroxide, hydroxyl radicals etc., may directly damage proteins, lipids and DNA, forming products that can be used as biomarkers of oxidative stress. In addition, oxidative stress through the activation of mitogen-activated protein kinase (MAPK) signaling as well as by increase of nuclear factor erythroid-2 related factors (NRF)-1 and -2 , and nuclear factor- $\kappa \mathrm{B}$ $(\mathrm{NF}-\kappa \mathrm{B})$ promotes inflammation (4) and modulates airway responses (5). Even, higher ROS may contribute to the induction of allergic asthma $(4,6)$.

Participation of various pathomechanisms results in a heterogeneity of clinical expressions of bronchial asthma and finally relates to the different response to administered therapy (1). Therefore, understanding the pathophysiology of the disease may be useful not only for developing and testing novel therapeutic strategies, but the precisely addressed therapeutic intervention may finally reduce also the proportion of patients not responding to the therapy.

Whereas the mobilization and activation of the cells in allergic inflammation and overproduction of ROS are the fundamental factors in the pathophysiology of the allergic

Address for correspondence

Daniela Mokra, MD., PhD., Department of Physiology, Jessenius Faculty of Medicine, Mala Hora 4, 03601 Martin, Slovakia e-mail: mokra@jfmed.uniba.sk 
asthma, the goal of this pilot study was to evaluate the mobilization of cells into the lungs and to determine the severity of oxidation processes induced by OVA-sensitization in a guinea-pig model of allergic asthma.

\section{METHODS}

The study protocol was approved by a local Ethics Committee at Jessenius Faculty of Medicine, Comenius University in Martin. In total, twelve healthy male guinea pigs (250-350 g) were used in the study, divided into two groups. There were no differences between the groups in age or body weight. Animals were kept in an animal house and had food and water ad libitum.

In six animals, model of allergic asthma was induced with ovalbumin allergen (Albumin from chicken egg white; Sigma-Aldrich, Germany). Other six animals served as non-sensitized (naïve) controls. Sensitization of animals with ovalbumin was performed during 14 days (7). Ovalbumin in a $1 \%$ concentration (10 $\mathrm{mg}$ of ovalbumin in $1 \mathrm{ml}$ ) was administered on the $1^{\text {st }}$ day of sensitization intraperitoneally $(0.5 \mathrm{ml})$ and subcutaneously $(0.5 \mathrm{ml})$, on the $3^{\text {rd }}$ day intraperitoneally $(1 \mathrm{ml})$. Ovalbumin challenge by the inhalation $(3 \mathrm{~min})$ was performed on the $14^{\text {th }}$ and $21^{\text {st }}$ day using an Aerosol Jet Nebulizer (HSE, Germany). Saline was given to the control (naïve) animals at the same dose and regimen as in OVA-sensitized ones. Animals were killed 30 min. after the second ovalbumin challenge.

After sacrificing the animal, sample of blood was taken and centrifuged at $3000 \mathrm{rpm}$ for 20 min. Blood plasma was stored at $-70{ }^{\circ} \mathrm{C}$ till biochemical analyses were made.

Left lungs were lavaged with saline $(2 \times 10 \mathrm{ml} / \mathrm{kg}$ b.w.). Total number of cells in BAL was evaluated microscopically and expressed per $1 \mu \mathrm{l}$ of BAL fluid. BAL fluid was then centrifuged at $1500 \mathrm{rpm}$ for $10 \mathrm{~min}$. In the sediment, differential leukocyte count was evaluated microscopically after staining by Pappenheim, as described previously (8).

Right lung tissue was homogenized, ECP, TAS and markers of lipid and protein oxidation (TBARS and lysine-LPO end-products) were determined (see below).

Lung tissue was washed and homogenized in $50 \mathrm{mmol}$ of phosphate buffer (pH 7.4) and $1 \mathrm{mmol}$ butylated hydroxytoluene in a ratio 1:5 using a homogenizer (Potter, B. Braun Melsungen A.G., Germany) at a temperature 0-4 ${ }^{\circ} \mathrm{C}$. Protein assay was performed by a method of Lowry et al. (9). For dilution of the homogenate, 1\% sodium dodecyl sulfate was used. Concentration was calculated using a bovine serum albumine as a standard.

Fluorescence measurements were performed in a solution containing $50 \mu \mathrm{g}$ of membrane protein per $\mathrm{ml}, 10 \mathrm{mmol} / 1 \mathrm{HEPES}, 100 \mathrm{mmol} / 1 \mathrm{KCl}, \mathrm{pH} 7.0$ at $25{ }^{\circ} \mathrm{C}$ using spectrofluorimeter (RF-540, Shimadzu, Japan). Fluorescence emission spectra (380-440 nm, slit width $5 \mathrm{~nm}$ ) of dityrosine, a product of tyrosine oxidation, were measured at excitation wavelength $325 \mathrm{~nm}$ (slit width $5 \mathrm{~nm}$ ) (10). Emission spectra (from 425 to $480 \mathrm{~nm}$, slit width $5 \mathrm{~nm}$ ) of lysine conjugates with lipid peroxidation (LPO) end-products were recovered at excitation of $365 \mathrm{~nm}$ (5 nm slit width). Excitation spectra (from 325 to $380 \mathrm{~nm}, 5 \mathrm{~nm}$ slit width) were measured at $440 \mathrm{~nm}$ (5 nm slit width) (11). Fluorescence intensity was expressed in arbitrary units (A.U.). Thiobarbituric acid-reactive substances (TBARS) in the lung homogenate were determined from the absorbance at $532 \mathrm{~nm}$ and expressed in nmol/mg protein (12).

Concentrations of eosinophil cationic protein (ECP) in the lung homogenate and in the blood plasma were evaluated by enzyme-linked immunosorbent assay (ELISA) using ECP kit (Diagnostics Development, Sweden). Quantification of total antioxidant status (TAS) in the lung homogenate and in the plasma was carried out using ABTS (2,2'-azino-di-[3-ethylbenzthiazoline sulphonate]) radical formation kinetics (Randox TAS kit, Randox Laboratories Ltd., UK) and were expressed in mmol/1.

Statistics: Statistical analysis was made by Systat for Windows. Because of relatively small number of animals in the groups and non-parametric distribution of some data, KruskalWallis test for between-group comparisons was used. A $P<0.05$ was considered statistically significant. 


\section{RESULTS}

\section{Cells in the BAL fluid and in the arterial blood}

OVA-sensitization elevated a total number of cells in the BAL fluid compared to naïve controls $\left(69.1 \pm 6.6 \times 10^{3} / \mu \mathrm{l}\right.$ in OVA-sensitized group vs. $9.0 \pm 1.5 \times 10^{3} / \mu 1$ in controls; $\left.P<0.01\right)$. In addition, OVA-sensitization increased percentages of both eosinophils $(P<0.01 ;$ Fig. 1$)$ and neutrophils $(P<0.05$; Fig. 2).

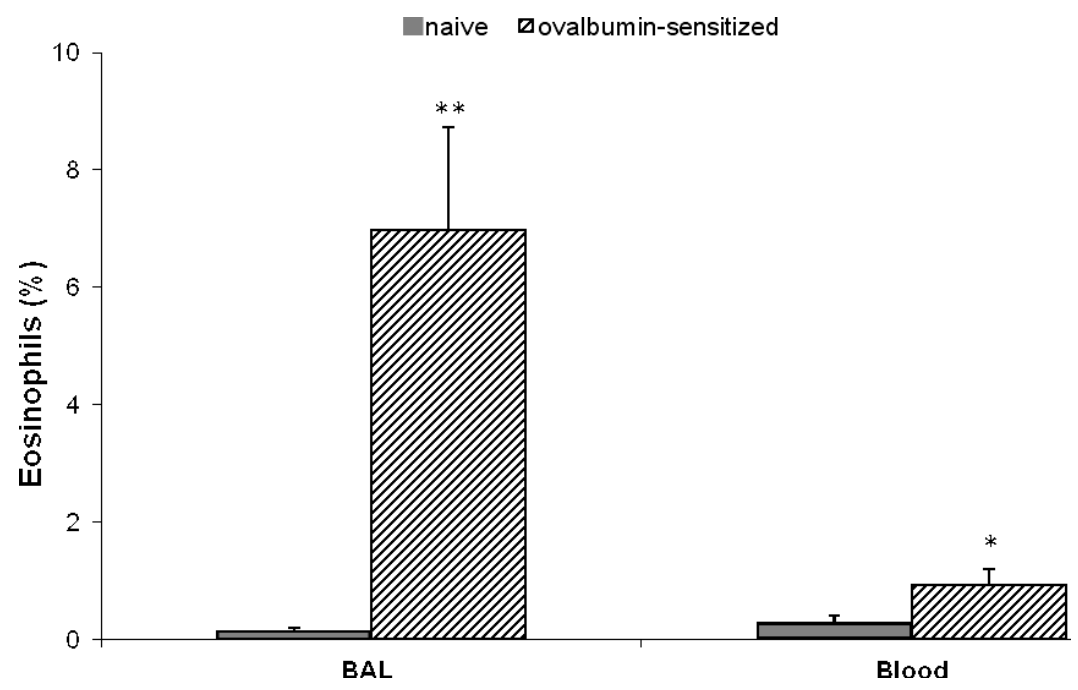

Fig. 1 Eosinophils (\%) in the BAL fluid and in the blood. For differences between OVA-sensibilized group vs. naïve animals: ${ }^{* *} P<0.01,{ }^{*} P<0.05$

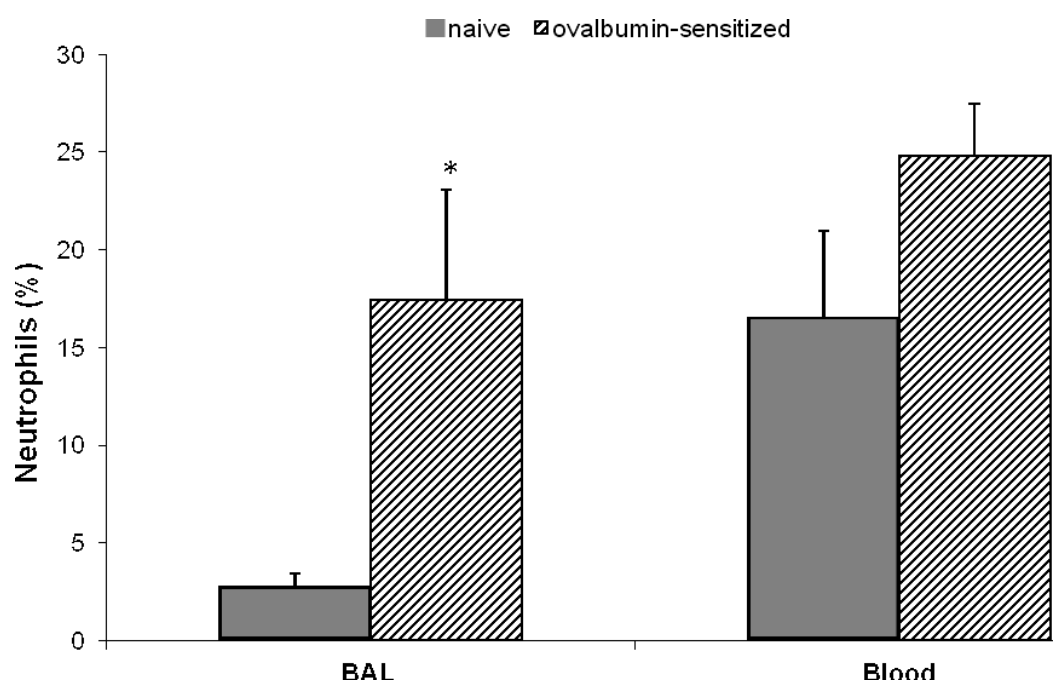

Fig. 2 Neutrophils (\%) in the BAL fluid and in the blood. For differences between OVA-sensibilized group vs. naïve animals: ${ }^{*} P<0.05$ 
Total number of leukocytes in the blood increased after OVA-sensitization compared to controls $\left(4.1 \pm 0.5 \times 10^{9} / 1\right.$ in OVA-sensitized group vs. $2.5 \pm 0.3 \times 10^{9} / 1$ in controls; $\left.P<0.05\right)$. Similarly, OVA-sensitization increased percentage of eosinophils $(P<0.05$; Fig. 1) and showed a trend to elevate also a percentage of neutrophils ( $P>0.05$; Fig. 2$)$.

\section{Markers of inflammation and oxidation}

OVA-sensitization slightly increased levels of eosinophil cationic protein (ECP) in the lung homogenate $(1.41 \pm 0.27 \mu \mathrm{g} / 1$ in OVA-sensitized group vs. $0.85 \pm 0.15 \mathrm{~g} / \mathrm{ml}$ in controls) and in the plasma $(2.10 \pm 0.29 \mu \mathrm{g} / 1$ in OVA-sensitized group vs. $1.21 \pm 0.36 \mu \mathrm{g} / 1$ in controls), however, these differences were not significant (both $P>0.05$ ).

In OVA-sensitized animals, higher concentrations of thiobarbituric acid-reactive substances (TBARS) and lysine-LPO end-products, markers of lipid and protein oxidation, were observed in the lung homogenate compared to non-sensitized controls (both $P<0.01$; Fig. 3A and 3B).
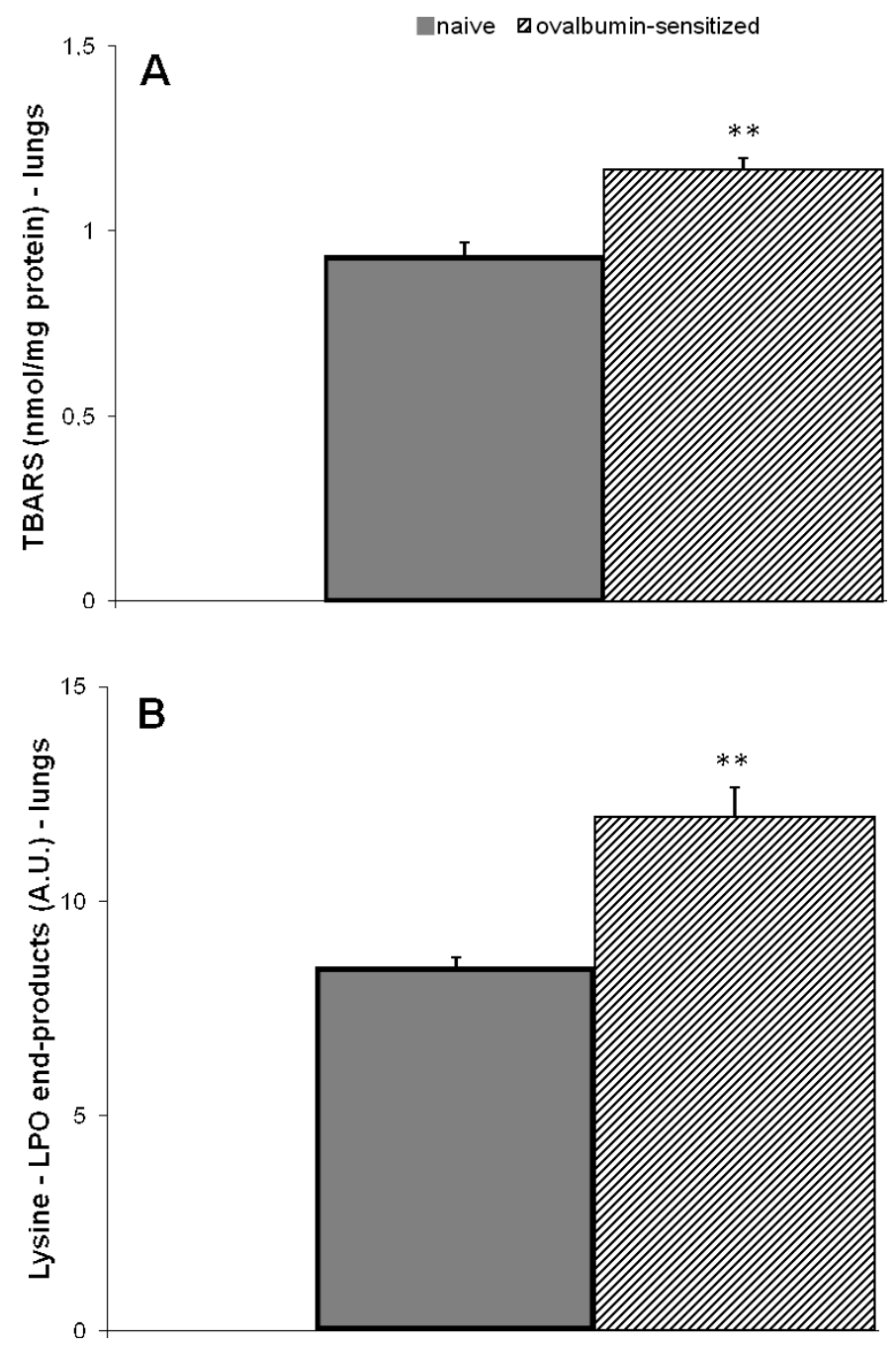

Fig. 3. Thiobarbituric acid-reactive substances (TBARS) (A) and lysine-lipoperoxidation (LPO) end-products (B) in the lung homogenate. For differences between OVA-sensitized group vs. naïve controls: ${ }^{* *} P<0.01$ 
Inversely to increased oxidation stress, total antioxidant status (TAS) decreased in the blood plasma $(P<0.05)$ and a trend to decrease TAS was observed also in the lung homogenate $(P>0.05$; Fig. 4).

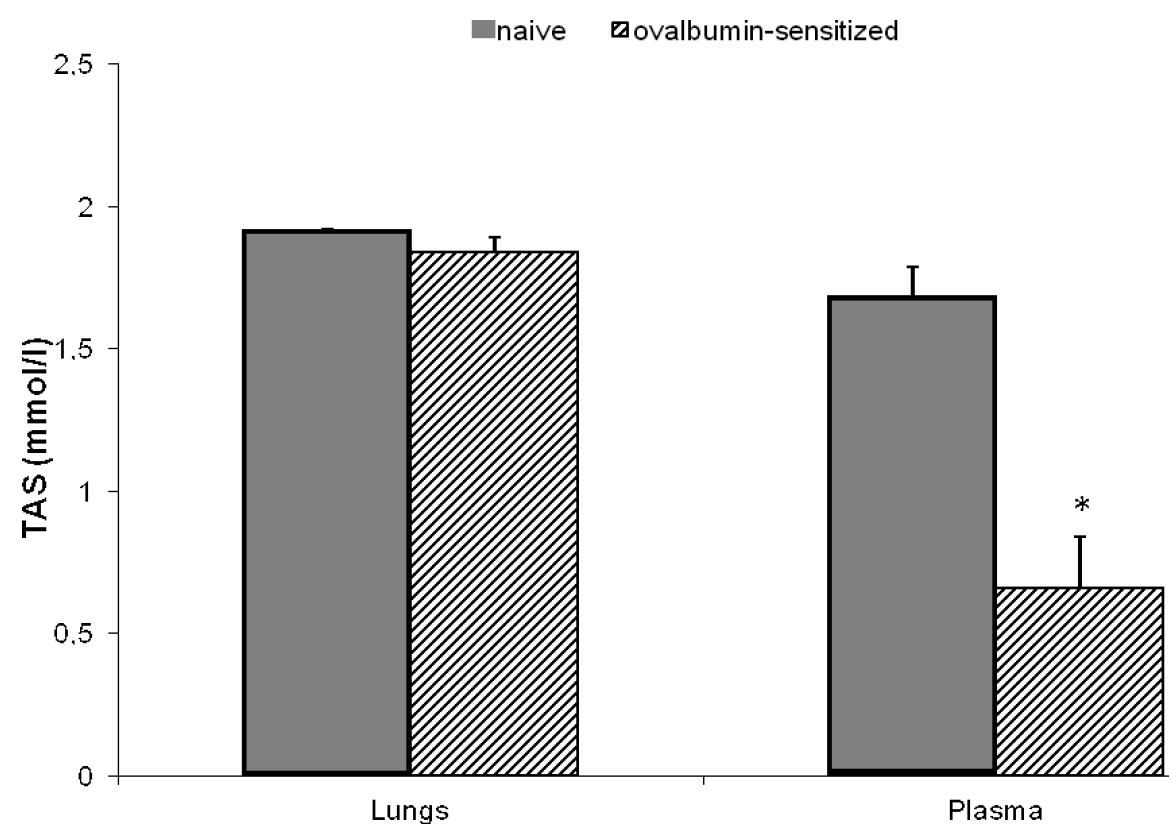

Fig. 4 Total antioxidant status (TAS) in the lung homogenate and in the blood plasma. For differences between OVA-sensitized group vs. naïve controls: ${ }^{*} P<0.05$.

\section{DISCUSSION}

Migration and activation of the inflammatory cells and abundant production of ROS are the fundamental factors in the pathophysiology of allergic asthma. Thus, the goal of this pilot study was to evaluate the mobilization of cells into the lungs and to determine the severity of oxidation processes induced by OVA-sensitization. In a guinea-pig model, OVA-sensitization elevated numbers of total leukocytes and percentages of eosinophils and neutrophils in the blood and increased total number of cells and percentages of eosinophils and neutrophils in the BAL fluid. Enhanced activation of cells, particularly of eosinophils, was confirmed by slightly increased levels of eosinophil cationic protein (ECP) in the lung and plasma. In addition, OVA-sensitization increased generation of free radicals and forming byproducts of lipid and protein peroxidation, associated with reduced total antioxidant status (TAS) in the lungs and in the blood plasma.

OVA-sensitization is a generally accepted method of preparation of an experimental model of airway inflammation and hyperreactivity, resembling the changes in bronchial asthma (3, $5,7)$. Several species of rodents may be used for this purpose, however, a guinea pig model has been well established.

Ovalbumin-sensitization in guinea pigs caused an increase in total circulating leukocytes with a particular increase in eosinophils and neutrophils compared to non-sensitized controls. This may be attributed to demarginalization and release of the mentioned types of leukocytes from the stores in the spleen and other organs. Anyway, we cannot exclude also 
the production de novo in the bone marrow, as the OVA-sensitization is a long-term process. An increase in circulating leukocytes goes hand-in-hand with an increase in a number of cells in the BAL fluid. Similarly to blood, a particular increase was found for percentages of eosinophils and neutrophils. It is known that these two types of phagocyting microcytes are able within a very short time to migrate from the circulation into the tissue. The process of their activation is associated with an increased production of a variety of bioactive substances $(1,13)$. Eosinophils are a rich source of granule basic proteins, but they generate prostacyclin, cysteinyl leukotrienes, reactive oxygen species, growth factors and a wide range of cytokines and chemokines, as well. Despite eosinophils produce lower amounts of cytokines and free radicals than the other leukocytes, they play an essential role in the immune response, airway hyperreactivity, airway remodelling and mucus production in allergic asthma $(1,13)$. On the other hand, neutrophils are potent producers of reactive oxygen species, however, their role as the secondary effector cells in a „classical“ allergic asthma of Th2-type, particularly mild-to-moderate asthma, is questionable (1). Nevertheless, neutrophilic inflammation was noted in patients with severe asthma and during acute exacerbations, as well as in patients treated with glucocorticoids $(1,14)$. Finding increased neutrophils in an absence of eosinophils suggests that the disease becomes more aggressive and chronic, representing non-eosinophilic asthma of Th1-type (1). More prominent increase in the eosinophils in our study is, definitively, in accordance with characteristics of the used model of allergic asthma.

To evaluate activation of eosinophils, ECP concentrations were determined in the lungs as well as in the blood plasma. Despite we found a slight increase in both lungs and plasma, the differences to the controls were not significant. This finding may be related to small number of animals included in the groups. In addition, we may speculate that although ECP increases in allergic asthma in humans (15), it might be not enough sensitive indicator of the eosinophil activation in this animal model of asthma and any other marker may be more appropriate, e.g. eotaxin etc.

Nevertheless, activation of eosinophils and neutrophils was satisfactory confirmed by finding increased concentrations of by-products of lipid and protein oxidation. A product of lipoperoxidation, malonyldialdehyde, may react with free sulfhydryl groups of cysteine or amino groups of lysine and generate complexes reacting with thiobarbituric acid, creating thiobarbituric acid-reactive substances (TBARS). In this study, higher concentrations of TBARS and lysine-LPO products have proven an overproduction of free radicals after ovalbumin-sensitization in guinea-pigs. Similarly, intensive oxidation stress characterized by increased TBARS was found in asthmatic children compared with healthy controls (16).

Inversely to enhanced generation of free radicals, a total antioxidant status (TAS) representing a cumulative action of all antioxidant systems in the plasma or lung tissue, decreased after OVA-sensitization. Similarly to our results, lower plasma total antioxidant capacity negatively correlated with oxidation markers were found also in patients with asthma, particularly in acute exacerbations (17). In addition, finding of reduced TAS levels in plasma suggests significant inflammatory and oxidation changes resulting from OVA-sensitization also on the systemic level.

In conclusion, OVA-sensitization in guinea pigs increased a number of circulating leukocytes in blood, particularly the percentages of eosinophils and neutrophils. Migration of these cells into the lungs was associated with a slight increase in eosinophil cationic protein, and significant overproduction of free radicals expressed by elevated levels of by-products of lipid and protein oxidation, and reduced total antioxidant status in the plasma.

\section{REFERENCES}

1. Holgate ST. Pathogenesis of asthma. Clin Exp Allergy 2008; 38: 872-897.

2. Rahman I, Biswas SK, Kode A. Oxidant and antioxidant balance in the airways and airway diseases. Eur J Pharmacol 2006; 533: 222-239. 
3. Park CS, Kim TB, Lee KY, et al. Increased oxidative stress in the airway and development of allergic inflammation in a mouse model of asthma. Ann Allergy Asthma Immunol 2009; 103: 238-247.

4. Ciencewicki J, Trivedi S, Kleeberger SR. Oxidants and the pathogenesis of lung diseases. J Allergy Clin Immunol 2008; 122: 456-468.

5. Talati M, Meyrick B, Peebles RS Jr., et al. Oxidant stress modulates murine allergic airway responses. Free Radic Biol Med 2006; 40: 1210-1219.

6. Cho YS, Moon HB. The role of oxidative stress in the pathogenesis of asthma. Allergy Asthma Immunol Res 2010; 2: 183-187.

7. Mokry J, Nosalova G. Evaluation of the cough reflex and airway reactivity in toluene- and ovalbumin-induced airway hyperresponsiveness. J Physiol Pharmacol 2007; 58 Suppl 5: 419-426.

8. Mokra D, Drgova A, Mokry J, et al. Combination of budesonide and aminophylline diminished acute lung injury in animal model of meconium aspiration syndrome. J Physiol Pharmacol 2008;59 Suppl 6: 461-471.

9. Lowry OH, Rosebrough NJ, Farr AL, Randall RJ. Protein measurement with the Folin phenol reagent. J Biol Chem 1951; 193: 265-275.

10. Giulivi C, Davies KJ. Dityrosine: a marker for oxidatively modified proteins and selective proteolysis. Methods Enzymol 1994; 233: 363-371.

11. Dousset N, Ferretti G, Taus M, Valdiguiè P, Curatola G. Fluorescence analysis of lipoprotein peroxidation. Methods Enzymol 1994; 233: 459-469.

12. Das DK. Cellular, biochemical and molecular aspects of reperfusion injury. Ann NY Acad Sci 1994; 723: 118124.

13. Stone KD, Prussin C, Metcalfe DD. IgE, mast cells, basophils, and eosinophils. J Allergy Clin Immunol 2010; 125: S73-S80.

14. Nguyen LT, Lim S, Oates T, Chung KF. Increase in airway neutrophils after oral but not inhaled corticosteroid therapy in mild asthma. Respir Med 2005; 99: 200-207.

15. Jahnz-Rózyk K, Plusa T, Mierzejewska J. Eotaxin in serum of patients with asthma or chronic obstructive pulmonary disease: relationship with eosinophil cationic protein and lung function. Mediators Inflamm 2000; 9: $175-179$

16. Babusikova E, Jesenak M, Kirschnerova R, Banovcin P, Dobrota D. Association of oxidative stress and GST-T1 gene with childhood bronchial asthma. J Physiol Pharmacol 2009; 60 Suppl 5: 27-30.

17. Nadeem A, Raj HG, Chhabra SK. Increased oxidative stress in acute exacerbations of asthma. J Asthma 2005; 42: $45-50$.

\section{Acknowledgement}

The study was supported by Project "Center of Experimental and Clinical Respirology II", co-financed from EU sources, and by Grant VEGA No. 1/0030/11.

Received: March, 28, 2012

Accepted: June, 20, 2012 